\title{
An IQC Approach to Robust Estimation against Perturbations of Smoothly Time-Varying Parameters
}

\author{
Joost Veenman* and Hakan Köroğlu* and Carsten W. Scherer* \\ *Delft Center for Systems and Control, Delft University of Technology \\ Mekelweg 22628 CD Delft The Netherlands \\ Phone : $+31(0) 1527\{87171,85899\}$ Fax : +31(0)152786679 \\ E-Mail : joostveenman@gmail.com, \{h.koroglu,c.w.scherer\}@tudelft.nl
}

\begin{abstract}
In this paper the design of robust $\mathscr{H}_{2}$-estimators for the class of linear systems that depend rationally on ratebounded uncertain time-varying parameters is addressed. The uncertainties are characterized by dynamic IQCs and new LMI conditions are formulated that allow for a nontrivial extension of the estimation problem with a weighting filter at the output. In order to show the power of the dynamic IQC approach, a numerical example is included in which the result is compared with alternative approaches based on parameter-dependent Lyapunov functions. The effectiveness of the additional weighting filter is illustrated in a second numerical example.
\end{abstract}

\section{INTRODUCTION}

During the last decade, the robust $\mathscr{H}_{2}$-estimator synthesis problem for linear systems that are subject to white-noise disturbances and norm-bounded parameter uncertainties has been studied intensively. The problem can be formulated as the design of a linear time-invariant estimator that guarantees a norm-bound on the asymptotic variance of the estimation error. Some early works, [1], [2], proposed solutions based on Riccati equations and later in [3], [4] methods based on LMI techniques were introduced for systems with parametric uncertainties that can be captured by a polytopic region. It is well known that these methods might be rather conservative since the uncertain parameters are allowed to vary arbitrarily fast. For this reason, there have been various attempts to reduce conservatism by using parameter-dependent Lyapunov functions (PDLF). In [5] and [6], methods were suggested for systems that depend affinely on uncertain time-invariant parameters. Only recently, in [7], [8], [9], these methods were generalized to the case in which the parameters are allowed to be time-varying. Despite the fact that the results were considerably improved, they all still might yield conservative results. A second drawback is that the system matrices are restricted to depend affinely on the uncertain parameters.

An alternative framework for stability and performance analysis is the dynamic integral quadratic constraint (IQC) approach, which was introduced in [10]. IQCs are very useful in describing different types of uncertainties. One could think, e.g., of uncertain time-delays, multiple static nonlinearities or uncertain LTI dynamics. Our special attention is dedicated to IQC tests for smoothly time-varying parametric uncertainties with bounded rates-of-variation, as was shown in [11], [12], and as was recently extended in [13] and [14]. Until very recently, the dynamic IQC-framework was mostly suitable for analysis purposes only. The essential difficulty in synthesis based on dynamic IQCs was the characterization of stability of the closed-loop system. Recently this problem was completely resolved in [15]. A necessary and sufficient positivity condition was derived that can be imposed on the synthesis LMIs in order to enforce stability of the corresponding closed-loop system. It was also shown in [15] that this result yields a complete solution for the $\mathscr{L}_{2}$-gain and $\mathscr{H}_{2}$-estimator synthesis problems if the uncertainties are described by dynamic IQCs.

The first goal of this paper is to merge the new results of [15] with the special IQC test for uncertain smoothly time-varying parameters from [13] and to show that we can systematically reduce conservatism with respect to the PDLF based approaches for a broader class of systems. The second goal is to show that the estimator synthesis problem from [15] can be further generalized to the use of a weighting filter at the output. With this filter, we can improve performance in a desired frequency band of interest at the cost of a possible degradation of performance outside the desired frequency band. After introducing some preliminaries in Section II, we formally state the problem in Section III. Then in Section IV, we give a short recap of IQC theory and describe the special IQC test for smoothly time-varying parameter uncertainties as it was presented in [13]. This is then merged with an IQC test for $\mathscr{H}_{2}$-performance. In Section V, we state and prove the main result of this paper, namely, a solution in terms of LMI conditions for the robust estimation problem with a weighting filter at the output for systems that depend rationally on uncertain smoothly time-varying parameters. In Section VI, two numerical examples are given. In the first example, we show that it is indeed possible to further reduce conservatism with respect to the PDLF approaches that are known from the literature. The second example illustrates the effect of using a weighting filter that emphasizes a certain frequency band in the estimation error. We conclude the paper with some final remarks. 


\section{Notation AND PRELIMINARIES}

The symbol $\mathscr{L}_{2 \mathrm{e}}^{n}[0, \infty)$ is used to denote the extended space of $\mathbb{R}^{n}$-valued functions that are square integrable on finite intervals. $\mathscr{L}_{2}^{n}[0, \infty) \subset \mathscr{L}_{2 \mathrm{e}}^{n}[0, \infty)$ represents the space of $\mathbb{R}^{n}$-valued functions, $g:[0, \infty) \rightarrow \mathbb{R}^{n}$, of finite energy $\|g\|^{2}:=\langle g, g\rangle:=\int_{0}^{\infty}|g(t)|^{2} d t$. The Fourier transform of $g(t)$ is denoted by $\hat{g}(i \omega)$. By an operator we mean a map $G: \mathscr{L}_{2 \mathrm{e}}^{a}[0, \infty) \rightarrow \mathscr{L}_{2 \mathrm{e}}^{b}[0, \infty)$ that takes one $\mathscr{L}_{2 \mathrm{e}}[0, \infty)$ space into another one. $\mathscr{R} \mathscr{L}_{\infty}^{m \times n}\left(\mathscr{R} \mathscr{H}_{\infty}^{m \times n}\right)$ will be used to denote the space of all real-rational and proper (and stable) matrix functions that have no poles on the imaginary axis (closed right-half complex-plane).

We will view time-varying uncertain parameters as static linear time-varying (LTV) operators $\Delta \in \mathscr{L}_{\mathrm{s}}$ (acting by multiplication on signals), where $\mathscr{L}_{\mathrm{s}}:=\{\Delta:[0, \infty) \rightarrow \mathbb{R}\}$. The variation of $\Delta$ is defined by another static LTV operator $\mathscr{V}_{\Delta}=\frac{d}{d t} \Delta-\Delta \frac{d}{d t}$ acting on differentiable signals and being identical to multiplication with $\mathscr{V}_{\Delta}(t):=$ $d \Delta(t) / d t$ (assuming $\Delta(t)$ is differentiable). For a precise definition see [13]. The preceding concept of a timevarying operator and its rate-of-variation motivates to introduce the concept of the so called region-of-variation $(\mathrm{ROV}) \mathscr{R}:=\left\{\left(\Delta(t), \mathscr{V}_{\Delta}(t)\right), t \in[0, \infty)\right\}$ where we assume $\mathscr{R}$ to be compact and star-shaped with star-center zero, i.e. $\tau \mathscr{R} \subset \mathscr{R}, \forall \tau \in[0,1]$. We describe the set of static LTV operators that vary in this region as $\mathscr{L}_{\mathscr{R}}:=$ $\left\{\Delta \in \mathscr{L}_{\mathrm{s}}:\left(\Delta(t), \mathscr{V}_{\Delta}(t)\right) \in \mathscr{R}, \forall t \in[0, \infty)\right\}$

\section{Problem Formulation}

Consider the system interconnection in Figure 1 where $G \in \mathscr{R} \mathscr{H}_{\infty}$ represents a nominal and stable LTI system that admits a minimal realization of the form

$$
G:=\left(\begin{array}{cc}
G_{q p} & G_{q w} \\
G_{z p} & G_{z w} \\
G_{y p} & G_{y w}
\end{array}\right)=\left[\begin{array}{c|cc}
A & B_{p} & B_{w} \\
\hline C_{q} & D_{q p} & D_{q w} \\
C_{z} & D_{z p} & D_{z w} \\
C_{y} & D_{y p} & D_{y w}
\end{array}\right],
$$

with $A \in \mathbb{R}^{n \times n}, B_{p} \in \mathbb{R}^{n \times n_{p}}, B_{w} \in \mathbb{R}^{n \times n_{w}}, C_{q} \in$ $\mathbb{R}^{n_{q} \times n}, C_{z} \in \mathbb{R}^{n_{z} \times n}$ and $C_{y} \in \mathbb{R}^{n_{y} \times n}$. The plant is subject to perturbations of static LTV operators $\Delta \in \Delta \subset$ $\mathscr{L}_{s}^{n_{p} \times n_{q}}$, with the set $\Delta$ representing the uncertainty. For notational simplicity, we consider a single but repeated LTV uncertainty $\Delta=\Delta_{r}:=I_{r} \otimes \delta \in \Delta$ where $\boldsymbol{\Delta}=$ $\left\{\left(I_{r} \otimes \delta\right): \delta \in \mathscr{L}_{\mathscr{R}}\right\}, \mathscr{R} \subset \mathbb{R}^{2} . \Delta_{r}$ interacts with $G$ through a linear fractional transformation for which we require the inverse $\left(I-D_{q p} \Delta_{r}\right)^{-1}$ to exist for all $\Delta_{r} \in \boldsymbol{\Delta}$. Since $D_{q p}$ and $\Delta$ are known this can be easily verified on beforehand.

The main goal in robust estimation is the synthesis of a stable LTI system $E:=C_{E}\left(s I-A_{E}\right)^{-1} B_{E}+D_{E}$ that dynamically processes the measurement $y \in \mathbb{R}^{n_{y}}$ to provide an estimate $z_{\mathrm{e}} \in \mathbb{R}^{n_{z}}$ of the signal $z \in \mathbb{R}^{n_{z}}$ with a guaranteed performance level in the $\mathscr{H}_{2}$-sense. For general operators, there are several definitions of the $\mathscr{H}_{2}$ norm, which are not necessarily equivalent (see e.g [16] and references therein). We will employ a signal based interpretation of the $\mathscr{H}_{2}$-norm. For this purpose we define the real vector $w^{\eta}=\left(\begin{array}{lllllll}0 & \cdots & 0 & 1 & 0 & \cdots & 0\end{array}\right)^{T} \in \mathbb{R}^{n_{w}}$

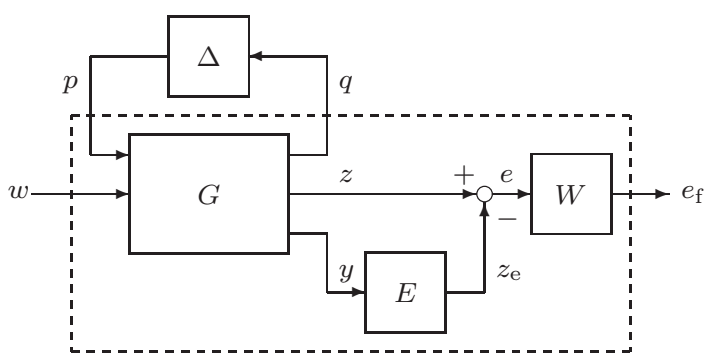

Fig. 1. Robust estimation problem.

with "1" being located at the $\eta^{t h}$ position. Let $e_{\mathrm{f}}^{\eta}$ be the response of the system to an impulsive input in the direction of $w^{\eta}$. We say that the squared $\mathscr{H}_{2}$-norm of the map $w \rightarrow e_{\mathrm{f}}$ is less then $\gamma>0$ if we have

$$
\sum_{\eta=1}^{n_{w}}\left\|e_{\mathrm{f}}^{\eta}\right\|_{2}^{2}<\gamma, \forall \Delta_{r} \in \Delta \text {. }
$$

In this paper we extend the robust estimation problem from [15] with a given stable weighting filter $W:=$ $C_{W}\left(s I-A_{W}\right)^{-1} B_{W}+D_{W}$, where $A_{W} \in \mathbb{R}^{n_{W} \times n_{W}}$, $B_{W} \in \mathbb{R}^{n_{W} \times n_{z}}, C_{W} \in \mathbb{R}^{n_{z} \times n_{W}}$. With this filter we can emphasize a certain frequency band of interest. Then we can improve performance within the frequency band of interest at the cost of a possible degradation of performance outside the frequency band of interest. Let us now formally state the robust estimation problem:

Problem 1: Given the plant $G$, the weighting filter $W$ and the compact region $\mathscr{R}$, design a stable LTI filter $E$ such that for all $\Delta_{r} \in \boldsymbol{\Delta}$ the squared $\mathscr{H}_{2}$-norm from $w \rightarrow e_{\mathrm{f}}$ is less then $\gamma>0$.

\section{Robust Stability And $\mathscr{H}_{2}$-Performance FOR LTV-SYSTEMS}

Recall that an uncertainty $\Delta \in \Delta$ is said to satisfy the IQC defined by the multiplier $\Pi \in \mathscr{R} \mathscr{L}_{\infty}^{\left(n_{q}+n_{p}\right) \times\left(n_{q}+n_{p}\right)}$ if the following condition holds true:

$$
\left\langle\left(\begin{array}{c}
q \\
\Delta q
\end{array}\right), \Pi\left(\begin{array}{c}
q \\
\Delta q
\end{array}\right)\right\rangle \geq 0, \forall q \in \mathscr{L}_{2}^{n_{q}} .
$$

In applications one constructs a whole family of multipliers that are parameterized as $\Pi=\Psi^{*} \mathbf{P} \Psi$ with a suitable set of symmetric matrices $\mathbf{P}$ and with a typically tall $\Psi \in$ $\mathscr{R} \mathscr{H}_{\infty}^{n_{\psi} \times n_{p}}$ such that the IQC holds for all $\Pi \in \Pi$ and all $\Delta \in \Delta$. It is then well known from [10] that stability of the feedback interconnection $G_{q p}$ and $\Delta$ amounts to verifying whether the inverse $\left(I-D_{q p} \Delta\right)^{-1}$ exists for all $\Delta \in \Delta$ and whether there exists an $\epsilon>0$ and a $P=P^{T} \in \mathbf{P}$ for which

$$
\left(\begin{array}{c}
G_{q p}(i \omega) \\
I
\end{array}\right)^{*} \Psi(i \omega)^{*} P \Psi(i \omega)\left(\begin{array}{c}
G_{q p}(i \omega) \\
I
\end{array}\right) \preccurlyeq-\epsilon I, \forall \omega \in \mathbb{R} .
$$

Since we are concerned with LTV perturbations we employ the IQC-test from [13]. Let us therefore consider the following variant of the swapping lemma:

Lemma 1 (Swapping Lemma): Consider the perturbations $\Delta_{r}:=I_{r} \otimes \delta, \Delta_{l}:=I_{l} \otimes \delta, \Delta_{k}:=I_{k} \otimes \delta$ and the transfer 
matrix $H \in \mathscr{R} \mathscr{H}_{\infty}^{l \times r}$, and let $H_{B}$ and $H_{C}$ be defined based on a minimal realization of $H$ as

$$
H=\left[\begin{array}{c|c}
A_{H} & B_{H} \\
\hline C_{H} & D_{H}
\end{array}\right], H_{B}=\left[\begin{array}{c|c}
A_{H} & B_{H} \\
\hline I & 0
\end{array}\right], H_{C}=\left[\begin{array}{c|c}
A_{H} & I \\
\hline C_{H} & 0
\end{array}\right],
$$

where $A_{H} \in \mathbb{R}^{n_{H} \times n_{H}}, B_{H} \in \mathbb{R}^{n_{H} \times r}$ and $C_{H} \in \mathbb{R}^{n_{H} \times l}$. Then we have that

$$
\underbrace{\left(\begin{array}{cc}
H & H_{C} \\
0 & I
\end{array}\right)}_{H_{\mathrm{le}}} \underbrace{\left(\begin{array}{c}
\Delta_{r} \\
\mathscr{V}_{\Delta_{k}} H_{B}
\end{array}\right)}_{\Delta_{\mathrm{re}}}=\underbrace{\left(\begin{array}{cc}
\Delta_{l} & 0 \\
0 & \mathscr{V}_{\Delta_{k}}
\end{array}\right)}_{\Delta_{\mathrm{le}}} \underbrace{\left(\begin{array}{c}
H \\
H_{B}
\end{array}\right)}_{H_{\mathrm{re}}} .
$$

It is now possible to arrive at the following test from [13], which basically is a reformulation of the test from [12].

Theorem 1: Let $G_{q p} \in \mathscr{R} \mathscr{H}_{\infty}^{n_{q} \times n_{p}}$ be an arbitrary stable transfer matrix and let us be given a compact region $\mathscr{R} \subset \mathbb{R}^{2}$. Then for all $\Delta_{r} \in \boldsymbol{\Delta}$ the feedback interconnection of $G_{q p}$ and $\Delta_{r}$ is stable if there exist a matrix $P=P^{T}$ and an $\epsilon>0$ such that with $\tilde{G}_{q p}:=\left(\begin{array}{ll}G_{q p} & 0_{r \times k}\end{array}\right)$ and

$$
\Pi=\Psi^{*} P \Psi=\left(\begin{array}{cc}
H_{\mathrm{re}} & 0 \\
0 & H_{\mathrm{le}}
\end{array}\right)^{*}\left(\begin{array}{cc}
P_{11} & P_{12} \\
P_{12}^{T} & P_{22}
\end{array}\right)\left(\begin{array}{cc}
H_{\mathrm{re}} & 0 \\
0 & H_{\mathrm{le}}
\end{array}\right),
$$

the following FDI and LMI respectively hold true:

$$
\begin{gathered}
\left(\begin{array}{c}
\tilde{G}_{q p}(i \omega) \\
I
\end{array}\right)^{*} \Pi(i \omega)\left(\begin{array}{c}
\tilde{G}_{q p}(i \omega) \\
I
\end{array}\right) \preccurlyeq-\epsilon I, \forall \omega \in \mathbb{R} \\
\left(\begin{array}{c}
I \\
\Theta(\delta, v)
\end{array}\right)^{T} P\left(\begin{array}{c}
I \\
\Theta(\delta, v)
\end{array}\right) \succcurlyeq 0, \forall(\delta, v) \in \mathscr{R},
\end{gathered}
$$

where $\Theta(\delta, v):=\operatorname{diag}\left(I_{l} \otimes \delta, I_{k} \otimes v\right)$.

Remark 1: In order to render condition (3) tractable, several relaxation schemes are suggested in [13]. In our numerical examples we used the zeroth order Pólya relaxation. Let us hence be given the set of vertices $\left\{\left(\delta^{i}, v^{i}\right)\right\}_{i=1}^{m} \in \mathbb{R}^{2}$ and let the polytopic region be defined as

$\mathscr{P}_{\left\{\left(\delta^{i}, v^{i}\right)\right\}_{i=1}^{m}}:=\left\{\sum_{i=1}^{m} \alpha_{i}\left(\delta^{i}, v^{i}\right): \sum_{i=1}^{m} \alpha_{i}=1, \alpha_{i} \in[0,1]\right\}$,

such that the ROV $\mathscr{R}$ is now defined through the given set of vertices with $\Theta^{i}:=\operatorname{diag}\left(I_{l} \otimes \delta^{i}, I_{k} \otimes v^{i}\right)$. Then (3) is satisfied if there exists a $P=P^{T}$ for which

$$
\left(\begin{array}{c}
I \\
\Theta^{i}
\end{array}\right)^{T} P\left(\begin{array}{c}
I \\
\Theta^{j}
\end{array}\right) \succcurlyeq 0, i=1, \ldots, m, j=i, \ldots, m .
$$

Remark 2: The size of $P$ heavily depends on the McMillan degree of $H$. For a basis function of the form $H(s)=$ $\left(1(s+\alpha)^{-1} \ldots(s+\alpha)^{-\mu}\right)^{T} \otimes I_{r}$, we can obtain a minimal realization as

$$
\left[\begin{array}{c|c}
A_{H} & B_{H} \\
\hline C_{H} & D_{H}
\end{array}\right]=\left[\begin{array}{ccccc|c}
-\alpha & 0 & \cdots & \cdots & 0 & 1 \\
1 & \ddots & \ddots & \ddots & \vdots & 0 \\
0 & \ddots & \ddots & \ddots & \vdots & \vdots \\
\vdots & \ddots & \ddots & \ddots & 0 & \vdots \\
0 & \cdots & 0 & 1 & -\alpha & 0 \\
\hline & & 0 & & & 1 \\
& & I_{\mu} & & & 0
\end{array}\right] \otimes I_{r} .
$$

For this realization we then have that $l=(\mu+1) r$ and $k=\mu r$.
In order to be able to establish a test for $\mathscr{H}_{2}$-performance as well, we introduce the following notation:

$$
\begin{aligned}
& \left(\begin{array}{ccc}
H_{\mathrm{re}} \tilde{G}_{q p} & H_{\mathrm{re}} G_{q w} & 0 \\
H_{1 \mathrm{e}} & 0 & 0 \\
W \tilde{G}_{z p} & W G_{z w} & -W \\
\tilde{G}_{y p} & G_{y w} & 0
\end{array}\right)=\left[\begin{array}{c|ccc}
\tilde{A} & \tilde{B}_{1} & \tilde{B}_{2} & \tilde{B} \\
\hline \tilde{C}_{1} & \tilde{D}_{11} & \tilde{D}_{12} & 0 \\
\tilde{C}_{2} & \tilde{D}_{21} & \tilde{D}_{22} & \tilde{E}_{2} \\
\tilde{C} & \tilde{F}_{1} & \tilde{F}_{2} & 0
\end{array}\right]= \\
& =\left[\begin{array}{c:c|ccc}
\tilde{A}_{11} & \tilde{A}_{12} & \tilde{B}_{11} & \tilde{B}_{12} & \tilde{B}_{13} \\
\hdashline 0 & \tilde{A}_{22} & \tilde{B}_{21} & \tilde{B}_{22} & 0 \\
\hline 0 & \tilde{C}_{11} & D_{11} & \tilde{D}_{12} & 0 \\
\tilde{C}_{12} & \tilde{C}_{22} & \tilde{D}_{21} & \tilde{D}_{22} & \tilde{E}_{2} \\
0 & \tilde{C}_{23} & \tilde{F}_{1} & \tilde{F}_{2} & 0
\end{array}\right]= \\
& {\left[\begin{array}{l:lll|lllll}
A_{W} & 0 & 0 & B_{W} C_{z} & B_{W} D_{z p} & 0 & B_{W} D_{z w} & -B_{W}
\end{array}\right]} \\
& =\left[\begin{array}{c:cccccc:c}
0 & A_{H} & 0 & B_{H} C_{q} & B_{H}^{-} \bar{D}_{q p}^{z p} & 0 & B_{H} D_{q w} & 0 \\
0 & 0 & A_{H} & 0 & B_{H} & I & 0 & 0 \\
0 & 0 & 0 & A & B_{p} & 0 & B_{w} & 0 \\
\hline 0 & C_{H} & 0 & D_{H} C_{q} & D_{H} D_{q p} & 0 & D_{H} D_{q w} & 0 \\
0 & I & 0 & 0 & 0 & 0 & 0 & 0 \\
0 & 0 & C_{H} & 0 & D_{H} & 0 & 0 & 0 \\
0 & 0 & 0 & 0 & 0 & I & 0 & 0 \\
\hdashline \bar{C}_{W} & 0 & 0 & D_{W} \bar{C}_{z} & \bar{D}_{W}^{-} \bar{D}_{z p} & 0 & D_{W} D_{z w} & -\bar{D}_{W} \\
\hdashline 0 & 0 & 0 & \bar{C}_{y} & \bar{D}_{y p}^{-} & 0 & \bar{D}_{y w} & 0
\end{array}\right]
\end{aligned}
$$

where $\tilde{G}_{q p}=\left(\begin{array}{ll}G_{q p} & 0\end{array}\right), \tilde{G}_{z p}=\left(\begin{array}{ll}G_{z p} & 0\end{array}\right), \tilde{G}_{y p}=\left(\begin{array}{ll}G_{y p} & 0\end{array}\right)$. It is now easily verified that the weighted system interconnected with the estimator admits the following realization:

$$
\left[\begin{array}{c|cc}
\mathcal{A} & \mathcal{B}_{1} & \mathcal{B}_{2} \\
\hline \mathcal{C}_{1} & \mathcal{D}_{11} & \mathcal{D}_{12} \\
\mathcal{C}_{2} & \mathcal{D}_{21} & \mathcal{D}_{22}
\end{array}\right]:=
$$

$\left[\begin{array}{cc|cc}\tilde{A}+\tilde{B} D_{E} \tilde{C} & \tilde{B} C_{E} & \tilde{B}_{1}+\tilde{B} D_{E} \tilde{F}_{1} & \tilde{B}_{2}+\tilde{B} D_{E} \tilde{F}_{2} \\ B_{E} \tilde{C} & A_{E} & B_{E} \tilde{F}_{1} & B_{E} \tilde{F}_{2} \\ \hline \tilde{C}_{1} & 0 & \tilde{D}_{11} & \tilde{D}_{12} \\ \tilde{C}_{2}+\tilde{E}_{2} D_{E} \tilde{C} & \tilde{E}_{2} C_{E} & \tilde{D}_{21}+\tilde{E}_{2} D_{E} \tilde{F}_{1} & \tilde{D}_{22}+\tilde{E}_{2} D_{E} \tilde{F}_{2}\end{array}\right]$.

Let us also introduce the symmetric matrix variable $\mathcal{X}$ and the selection matrix $\Phi$ which are respectively partitioned as $\mathcal{X}=\left(\begin{array}{ccc}X_{11} & \cdots & X_{15} \\ \vdots & \ddots & \vdots \\ X_{15}^{T} & \cdots & X_{55}\end{array}\right), \Phi^{T}=\left(\begin{array}{ccccc}I & 0 & 0 & 0 & 0 \\ 0 & I & 0 & 0 & 0 \\ 0 & 0 & 0 & I & 0 \\ 0 & 0 & 0 & 0 & I\end{array}\right)$,

where $\Phi$ is compatible with to the block structure of $\mathcal{X}$ and where the sizes of $X_{11} \cdots X_{55}$ are equal to the sizes of $A_{W}$, $A_{H}, A_{H}, A, A_{E}$ respectively. We now can state the following feasibility test for robust stability and $\mathscr{H}_{2}$-performance:

Theorem 2: There exists a stable estimator $E$ such that for all $\Delta_{r} \in \boldsymbol{\Delta}$ the interconnection of Figure 1 is stable and the squared $\mathscr{H}_{2}$-norm from $w \rightarrow e$ is rendered less then $\gamma>0$, if $\mathcal{D}_{21}=0$ and $\mathcal{D}_{22}=0$ and if there exist matrices $P=P^{T}, \mathcal{X}=\mathcal{X}^{T}$ and $Z=Z^{T}$ for which (3) as well as the following conditions hold true:

$$
\left(\begin{array}{cc}
I & 0 \\
\mathcal{X} \mathcal{A} & \mathcal{X B}_{1} \\
\mathcal{C}_{1} & \mathcal{D}_{11} \\
\mathcal{C}_{2} & \mathcal{D}_{21}
\end{array}\right)^{T} \underbrace{\left(\begin{array}{cccc}
0 & I & 0 & 0 \\
I & 0 & 0 & 0 \\
0 & 0 & P & 0 \\
0 & 0 & 0 & I
\end{array}\right)}_{\mathcal{M}(P)}\left(\begin{array}{cc}
I & 0 \\
\mathcal{X} \mathcal{A} & \mathcal{X} \mathcal{B}_{1} \\
\mathcal{C}_{1} & \mathcal{D}_{11} \\
\mathcal{C}_{2} & \mathcal{D}_{21}
\end{array}\right) \prec 0
$$




$$
\begin{aligned}
\text { Trace }(Z) & <\gamma, \\
\mathcal{B}_{2}^{T} \mathcal{X} \mathcal{B}_{2} & \prec Z, \\
\Phi^{T} \mathcal{X} \Phi & \succ 0 .
\end{aligned}
$$

Proof: Since $\mathscr{R}$ is star-shaped with center zero, (3) implies that the left-upper block of $P$ is positive semidefinite. With a simplified version of the recent result in [15] we infer that (8) and (5) imply stability of $\mathcal{A}$ and, with the KYP-lemma, the validity of (2), which in turn proves robust stability of the feedback interconnection of $G_{q p}$ and $\Delta_{r}$.

Now in order to prove robust $\mathscr{H}_{2}$-performance, let us describe the closed-loop trajectories of system (4) for an impulsive input in the direction of $w^{\eta}$ as

$$
\left(\begin{array}{c}
\dot{x}^{\eta} \\
\hline q_{\mathrm{f}}^{\eta} \\
p_{\mathrm{f}}^{\eta} \\
\hline e_{\mathrm{f}}^{\eta}
\end{array}\right):=\left(\begin{array}{cc}
\mathcal{A} & \mathcal{B}_{1} \\
\mathcal{C}_{1} & \mathcal{D}_{11} \\
\mathcal{C}_{2} & \mathcal{D}_{21}
\end{array}\right)\left(\begin{array}{c}
x^{\eta} \\
p_{\mathrm{e}}^{\eta}
\end{array}\right), x^{\eta}(0)=\mathcal{B}_{2} w^{\eta},
$$

where $x^{\eta}(t), p_{\mathrm{e}}^{\eta}(t)$ and $e_{\mathrm{f}}^{\eta}(t)$ are the $\eta^{t h}$ evolutions of respectively the closed-loop state $x$, the signal $p_{\mathrm{e}}$ which is the extension of the input-signal $p$ and the estimation error signal $e$. Then after having right- and left-multiplied (7) and (5) with $w^{\eta}$ and $\left(x^{\eta}(t)^{T}, p_{\mathrm{e}}^{\eta}(t)^{T}\right)^{T}$ and their transposes respectively, we can integrate from zero to infinity to infer

$$
\begin{array}{r}
\int_{0}^{\infty}\left(\begin{array}{l}
q_{\mathrm{f}}^{\eta}(t) \\
p_{\mathrm{f}}^{\eta}(t)
\end{array}\right)^{T} P\left(\begin{array}{c}
q_{\mathrm{f}}^{\eta}(t) \\
p_{\mathrm{f}}^{\eta}(t)
\end{array}\right) d t+\int_{0}^{\infty} e_{\mathrm{f}}^{\eta}(t)^{T} e_{\mathrm{f}}^{\eta}(t) d t \\
\leq x^{\eta}(0)^{T} \mathcal{X} x^{\eta}(0) \leq w^{\eta T} Z w^{\eta} .
\end{array}
$$

Now observe that the filtered signals $\hat{p}_{\mathrm{f}}$ and $\hat{q}_{\mathrm{f}}$ are respectively defined through the relations $\hat{p}_{\mathrm{f}}=H_{\mathrm{le}} \hat{p}_{\mathrm{e}}$ and $\hat{q}_{\mathrm{f}}=H_{\mathrm{re}} \hat{q}$ where $\hat{q}=\tilde{G}_{q p} \hat{p}_{\mathrm{e}}$. We can then invoke Parseval's theorem, in order to transform the first term of (10) into

$$
\int_{-\infty}^{\infty}\left(\begin{array}{c}
\hat{q}_{\mathrm{f}}^{\eta} \\
\hat{p}_{\mathrm{f}}^{\eta}
\end{array}\right)^{*} P\left(\begin{array}{c}
\hat{q}_{\mathrm{f}}^{\eta} \\
\hat{p}_{\mathrm{f}}^{\eta}
\end{array}\right) d \omega=\int_{-\infty}^{\infty}\left(\begin{array}{c}
\hat{q}^{\eta} \\
\hat{p}_{\mathrm{e}}^{\eta}
\end{array}\right)^{*} \Pi\left(\begin{array}{c}
\hat{q}^{\eta} \\
\hat{p}_{\mathrm{e}}^{\eta}
\end{array}\right) d \omega
$$

Since this is nonnegative for all $\Delta_{r} \in \boldsymbol{\Delta}$, we conclude

$$
\int_{0}^{\infty} e_{\mathrm{f}}^{\eta}(t)^{T} e_{\mathrm{f}}^{\eta}(t) d t-w^{\eta T} Z w^{\eta} \leq 0, \forall w^{\eta} .
$$

Summing over $\eta$ then leads to (1) thanks to (6).

\section{MAin Results}

In this section we present a solution in terms of LMIs for the robust $\mathscr{H}_{2}$-estimator synthesis problem, extended with a weighting filter at the output. Let us hence introduce the matrix variables

$$
\begin{gathered}
T_{1}=\left(\begin{array}{cc}
I & 0 \\
T_{12}^{T} & T_{22}
\end{array}\right), T_{2}=\left(\begin{array}{cc}
T_{11} & -T_{12} \\
0 & I
\end{array}\right), T_{3}=\left(\begin{array}{cc}
T_{11} & 0 \\
0 & T_{22}
\end{array}\right), \\
X=\left(\begin{array}{ccc}
X_{11} & \cdots & X_{14} \\
\vdots & \ddots & \vdots \\
X_{14}^{T} & \cdots & X_{44}
\end{array}\right), R=\left(\begin{array}{cccc}
0 & 0 & 0 & 0 \\
0 & 0 & 0 & 0 \\
0 & 0 & S & 0 \\
0 & 0 & 0 & 0
\end{array}\right),
\end{gathered}
$$

where $R=R^{T}$ is partitioned according to the block structure of $X=X^{T}$ and where the sizes of $T_{11}=T_{11}^{T}, T_{12}, T_{22}=T_{22}^{T}$ are equal to the sizes of $\tilde{A}_{11}, \tilde{A}_{12}, \tilde{A}_{22}$ respectively. Then we are ready to state the main result of this paper.
Theorem 3: There exists a stable estimator $E$ such that for all $\Delta_{r} \in \boldsymbol{\Delta}$ the interconnection of Figure 1 is stable and the squared $\mathscr{H}_{2}$-norm from $w$ to $e$ is rendered less than $\gamma>0$, if there exist matrices $\tilde{K}, L, \tilde{M}, N, P=P^{T}, R=R^{T}$, $X=X^{T}, T_{1}, T_{2}, T_{3}, Z=Z^{T}$, for which (3), (6) as well as the following conditions hold true:

$$
\begin{aligned}
& D_{H} D_{q w}=0, \quad D_{W}\left(D_{z w}-N D_{y w}\right)=0, \\
& \left(\begin{array}{ccc}
R-T_{3} & R-T_{1}^{T} & T_{1}^{T} \tilde{B}_{2}+\tilde{B} N \tilde{F}_{2} \\
R-T_{1} & R-X & X \tilde{B}_{2}+L \tilde{F}_{2} \\
\tilde{B}_{2}^{T} T_{1}+\tilde{F}_{2}^{T} N^{T} \tilde{B}^{T} & \tilde{B}_{2}^{T} X+\tilde{F}_{2}^{T} L^{T} & -Z
\end{array}\right) \prec 0, \\
& \mathcal{O}^{T} \mathcal{M}(P) \mathcal{O} \prec 0,
\end{aligned}
$$

where

$$
\mathcal{O}=\left(\begin{array}{ccc}
I & 0 & 0 \\
0 & I & 0 \\
T_{1}^{T} \tilde{A} T_{2}+\tilde{B} \tilde{M} & T_{1}^{T} \tilde{A}+\tilde{B} N \tilde{C} & T_{1}^{T} \tilde{B}_{1}+\tilde{B} N \tilde{F}_{1} \\
\tilde{K} & X \tilde{A}+L \tilde{C} & X \tilde{B}_{1}+L \tilde{F}_{1} \\
\tilde{C}_{1} & \tilde{C}_{1} & \tilde{D}_{1} \\
\tilde{C}_{2} T_{2}+\tilde{E}_{2} \tilde{M} & \tilde{C}_{2}+\tilde{E}_{2} N \tilde{C} & \tilde{D}_{2}+\tilde{E}_{2} N \tilde{F}_{1}
\end{array}\right) .
$$

With solutions of these LMIs, one can then construct

$$
Y=\left(\begin{array}{cc}
T_{11}+T_{12} T_{22}^{-1} T_{12}^{T} & -T_{12} T_{22}^{-1} \\
-T_{22}^{-1} T_{12}^{T} & T_{22}^{-1}
\end{array}\right),
$$

and non-singular $U$ and $V$ such that $X Y+U V^{T}=I$. With $\bar{K}=\tilde{K} T_{1}^{-1}-X \tilde{A} Y$ and $M=\tilde{M} T_{1}^{-1}$, the estimator realization matrices $A_{E}, B_{E}, C_{E}, D_{E}$ can then be chosen as

$$
\left(\begin{array}{cc}
A_{E} & B_{E} \\
C_{E} & D_{E}
\end{array}\right)=\left(\begin{array}{cc}
U & X \tilde{B} \\
0 & I
\end{array}\right)^{-1}\left(\begin{array}{cc}
\bar{K} & L \\
M & N
\end{array}\right)\left(\begin{array}{cc}
V^{T} & 0 \\
\tilde{C} Y & I
\end{array}\right)^{-1} .
$$

Proof: Let $\mathcal{R}:=\left(\begin{array}{cc}R & 0 \\ 0 & 0\end{array}\right)$ and observe that (by the elimination lemma) (8) is equivalent to the existence of a $R=R^{T}$ for which $\mathcal{X}-\mathcal{R} \succ 0$. Moreover, since $\mathcal{R} \mathcal{B}_{2}=0$, (7) can be equivalently rewritten as

$$
\left(\begin{array}{cc}
\mathcal{R}-\mathcal{X} & \mathcal{X} \mathcal{B}_{2} \\
\mathcal{B}_{2}^{T} \mathcal{X} & -Z
\end{array}\right) \prec 0
$$

Let us now perform the congruence transformation and the bijective variable transformation that are well known to linearize the nominal output-feedback control problem (see e.g. [17]). For this we introduce the new variables $K, L, M$ and $N$ which are defined through the following equality:

$$
\begin{aligned}
&\left(\begin{array}{cc}
K & L \\
M & N
\end{array}\right)=\left(\begin{array}{cc}
X \tilde{A} Y & 0 \\
0 & 0
\end{array}\right)+ \\
&+\left(\begin{array}{cc}
U & X \tilde{B} \\
0 & I
\end{array}\right)\left(\begin{array}{ll}
A_{E} & B_{E} \\
C_{E} & D_{E}
\end{array}\right)\left(\begin{array}{cc}
V^{T} & 0 \\
\tilde{C} Y & I
\end{array}\right), \\
& \text { where } \\
& \mathcal{X}=\left(\begin{array}{cc}
X & U \\
U^{T} & \bullet
\end{array}\right), \mathcal{X}^{-1}=\left(\begin{array}{cc}
Y & V \\
V^{T} & \bullet
\end{array}\right), \mathcal{Y}=\left(\begin{array}{cc}
Y & I \\
V^{T} & 0
\end{array}\right) .
\end{aligned}
$$

Then (17) and (5) can be equivalently rewritten as

$$
\left(\begin{array}{ccc}
Y R Y-Y & Y R-I & \tilde{B}_{2}+\tilde{B} N \tilde{F}_{2} \\
R Y-I & R-X & X \tilde{B}_{2}+L \tilde{F}_{2} \\
\tilde{B}_{2}^{T}+\tilde{F}_{2}^{T} N^{T} \tilde{B}^{T} & \tilde{B}_{2}^{T} X+\tilde{F}_{2}^{T} L^{T} & -Z
\end{array}\right) \prec 0,
$$


and

$$
\tilde{\mathcal{O}}^{T} \mathcal{M}(P) \tilde{\mathcal{O}} \prec 0,
$$

respectively, where

$$
\tilde{\mathcal{O}}=\left(\begin{array}{ccc}
I & 0 & 0 \\
0 & I & 0 \\
\tilde{A} Y+\tilde{B} M & \tilde{A}+\tilde{B} N \tilde{C} & \tilde{B}_{1}+\tilde{B} N \tilde{F}_{1} \\
K & X \tilde{A}+L \tilde{C} & X \tilde{B}_{1}+L \tilde{F}_{1} \\
\tilde{C}_{1} Y & \tilde{C}_{1} & \tilde{D}_{1} \\
\tilde{C}_{2} Y+\tilde{E}_{2} M & \tilde{C}_{2}+\tilde{E}_{2} N \tilde{C} & \tilde{D}_{2}+\tilde{E}_{2} N \tilde{F}_{1}
\end{array}\right) .
$$

Since (18) and (19) are not yet affine in all the variables, we perform the additional congruence transformation with $\mathcal{T}:=\operatorname{diag}\left(T_{1}, I\right)$ (with compatible dimensions of $I$ ) that was suggested in [18] and as employed in [19] in a different context. Then the following relations hold true:

$$
\begin{gathered}
Y T_{1}=T_{2}, T_{1}^{T} Y T_{1}=T_{3}, R Y T_{1}=R, \\
K T_{1}=\tilde{K}, \quad M T_{1}=\tilde{M}, T_{1}^{T} \tilde{B}=\tilde{B}, \tilde{C}_{1} Y T_{1}=\tilde{C}_{1}, \\
T_{1}^{T} \tilde{A} T_{2}=\left(\begin{array}{cc}
\tilde{A}_{11} T_{11} & \tilde{A}_{12}-\tilde{A}_{11} T_{12}+T_{12} \tilde{A}_{22} \\
0 & T_{22} \tilde{A}_{22}
\end{array}\right) .
\end{gathered}
$$

Observe that (20) is affine in the variables $T_{11}, T_{12}, T_{22}$ and that the second congruence transformation yields (13) and (14). Moreover, applying the two suggested congruence transformations to (8) yields

$$
\mathcal{T}^{T} \mathcal{Y}^{T}(\mathcal{X}-\mathcal{R}) \mathcal{Y} \mathcal{T}=\left(\begin{array}{cc}
T_{3}-R & T_{1}^{T}-R \\
T_{1}-R & X-R
\end{array}\right) \succ 0,
$$

which is implied by (13). Finally observe that $\mathcal{D}_{12}$ and $\mathcal{D}_{22}$ are zero if and only if (12) is satisfied.

Remark 3: By applying the Schur complement with respect to the lower right identity block of $\mathcal{M}(P)$, we can transform (14) into a genuine LMI.

Remark 4: Though we have shown that it is possible to extend the estimation problem of [15] by applying a weighting-filter at the output for the class of LTV uncertainties with bounded rates of variation, the result can be easily extended to any type of uncertainty that can be suitably described by IQCs of the form $\Pi=\Psi^{*} P \Psi$, with appropriate adaptation of the stability condition from [15].

Remark 5: It is straightforward to arrive at a solution in terms of LMIs for the robust $\mathscr{L}_{2}$-estimator synthesis problem. Instead of (1), we need to guarantee $\left\langle e_{\mathrm{f}}, e_{\mathrm{f}}\right\rangle \leq$ $\left(\gamma^{2}-\epsilon\right)\langle w, w\rangle$ for some $\epsilon>0$. Conditions (12), (6) and (13) can then be disregarded and we only need to extend (14) with the appropriate performance matrices and impose (1) to enforce closed-loop stability.

Remark 6: The matrix variable $S$ as well as the matrix variables $\tilde{K}$ and $\tilde{M}$ can be eliminated (especially for analysis purposes). According to our experience, though, elimination of $S$ might deteriorate a numerically reliable construction of the estimator.

\section{NUMERICAL EXAMPLES}

We will first show that we can reduce conservatism with respect to the approaches based on PDLFs. We especially focus on the recent results of [8], [9] where the proposed $\mathscr{H}_{2}$-filter design methods are based on PDLFs that depend quadratically on the uncertain parameters. It should be mentioned that the stochastic interpretation of the $\mathscr{H}_{2}$-norm, which is used in [7], [8], [9], is equivalent to the signal based interpretation we are using such that we are allowed to compare our results. We hence took the following numerical example from [8], [9]:

$$
\left(\begin{array}{l}
q \\
z \\
y
\end{array}\right)=\left[\begin{array}{cc|cc:cc}
0 & -1 & 0 & 0.3 & -2 & 0 \\
1 & -0.5 & 0 & 0 & 1 & 0 \\
\hline 1 & 0 & 0 & 0 & 0 & 0 \\
0 & 1 & 0 & 0 & 0 & 0 \\
\hdashline-\frac{1}{1}-0 & 0 & 0 & 0 & 0 \\
\hdashline-100 & 0 & 0 & 0 & 1
\end{array}\right]\left(\begin{array}{l}
p \\
w
\end{array}\right) .
$$

Figure 2 displays the squared $\mathscr{H}_{2}$-norm from $w \rightarrow e(W=I)$ for the plant (21) with $|\delta(t)| \leq 1.4$ and for increasing values of $\mu$ (length of basis) $\mu$ and $|v|$ (rate-bound) for the methods from [8], [9] and those in this paper. The lines "IQC, $\mu=0$ " and "PDLF 0" correspond to the standard quadratic stability tests where $\delta(t)$ is allowed to vary arbitrarily fast. The lines "PDLF 1" and "PDLF 2" represent the results of [8] and respectively use a Lyapunov function that depends affinely and quadratically on $\delta$. The line "PDLF 3" corresponds to the results of [9] and is not a complete solution in terms of LMIs, due to a bilinear term. Hence, for this example, the IQC approach clearly outperforms all the PDLF approaches for sufficiently large $\mu$.



Fig. 2. Numerical Example 1.

In our second example we illustrate the possibility to further improve the estimation results by employing a weighting filter at the output for the mass-spring-damper system that is displayed in Figure 3. Mass $m_{1}$ is disturbed by an external

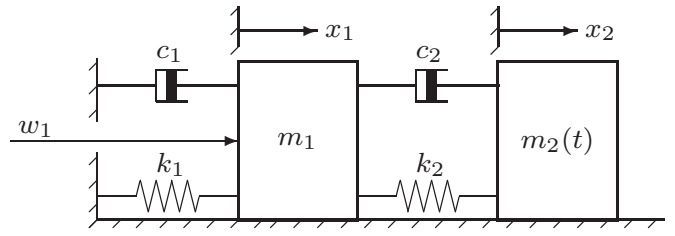

Fig. 3. Mass-spring-damper system with uncertain time-varying $m_{2}$.

force $w_{1}$ and mass $m_{2}$ is uncertain and time-varying. The objective is to estimate position $x_{2}$ based on the measurement $x_{1}$ which is corrupted by measurement noise $w_{2}$. Observe, with $k_{3}=k_{1}+k_{2}, c_{3}=c_{1}+c_{2}, p=\Delta q, \Delta=|\delta(t)|<1$ and 
$m_{2}=\hat{m}_{2}+W_{2} \delta(t)$, that $G$ can be written as

$$
\left(\begin{array}{l}
z \\
q \\
y
\end{array}\right)=\left[\begin{array}{cccc|cccc}
0 & 0 & 1 & 0 & 0 & 0 & 0 \\
0 & 0 & 0 & 1 & 0 & 0 & 0 \\
-\frac{k_{3}}{m_{1}} & \frac{k_{2}}{m_{1}} & -\frac{c_{3}}{m_{1}} & \frac{c_{2}}{m_{1}} & 0 & \frac{1}{m_{1}} & 0 \\
\frac{k_{2}}{\hat{m}_{2}} & -\frac{k_{2}}{\hat{m}_{2}} & \frac{c_{2}}{\hat{m}_{2}} & -\frac{c_{2}}{\hat{m}_{2}} & -\frac{W_{2}}{\hat{m}_{2}} & 0 & 0
\end{array}\right]\left(\begin{array}{l}
p \\
w
\end{array}\right) .
$$

For our illustration, we used the numerical values $m_{1}=10$, $\hat{m}_{2}=20, c_{1}=10, c_{2}=100, k_{1}=10, k_{2}=100, W_{2}=15$ and $\mathrm{a}$ parameter trajectory $\delta(t)=\sin \left(\frac{1}{4} t\right)$. By plotting $v=\dot{\delta}$ vs $\delta$ we observe that the ROV is an ellipse that can be covered by a polytope according to the left upper-plot of Figure 4. We used the weighting filter $W=\frac{2.5 s+5}{5 s+1}, \mu=3$, and a pulse signal as the input disturbance $w_{1}$ with an amplitude of 1000 that was repeated every $10 \mathrm{sec}$ with a random direction and a pulse width of $0.1 \%$ of the period. Finally we used a band-limited white-noise signal with variance of 0.01 for the disturbance input $w_{2}$. In the lower plot of Figure 4, the estimation error is shown for respectively the nominal design (i.e. $\delta \equiv 0$ ), the robust design and the robust design with a weighting filter at the output. Clearly the estimator that was designed with the weighting filter at the output leads to an error with smaller low frequency content.
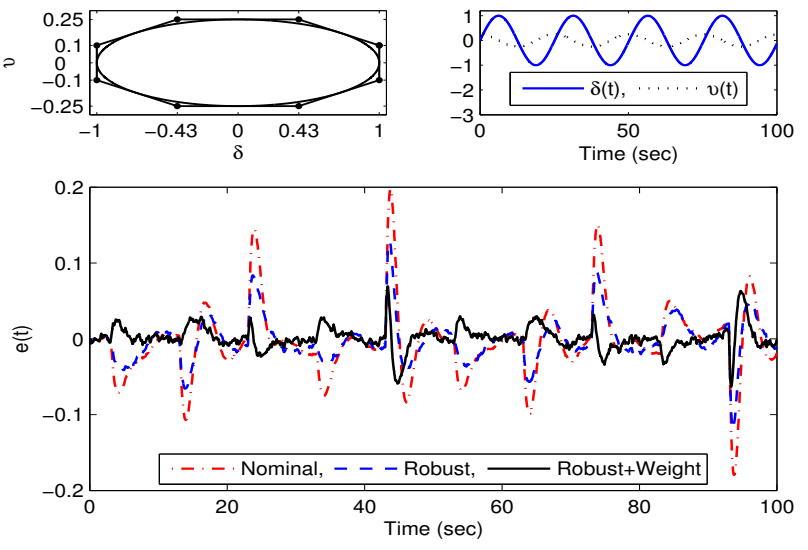

Fig. 4. Estimation error $e$ for sinusoidal perturbations $\delta(t)$.

\section{CONCLUding REMARKS}

We have given an LMI solution for an extension of the robust $\mathscr{H}_{2}$-estimator synthesis problem with a weighting filter at the output, and for systems that depend rationally on smoothly time-varying parameter uncertainties which are characterized with dynamic IQCs. Generalizations to any type of uncertainties that can be suitably described by dynamic IQCs are straightforward, once the IQC multipliers are factorized in the form assumed in this paper. Though the results were formulated for $\mathscr{H}_{2}$-estimation, the technique readily extends to $\mathscr{L}_{2}$-gain performance. We illustrated through two numerical examples how to systematically reduce conservatism with respect to approaches based on parameter dependent Lyapunov functions and how to benefit from an additional weighting filter at the performance output.

\section{ACKNOWLEDGEMENT}

The second and third authors are supported by the Technology Foundation STW, applied science division of NWO and the technology programme of the Ministry of Economic Affairs.

\section{REFERENCES}

[1] U. Shaked and C. E. de Souza, "Robust minimum variance filtering," IEEE Transactions on Signal Processing, vol. 43, pp. 2474-2483, 1995.

[2] Y. Theodor and U. Shaked, "Robust discrete-time minimum-variance filtering," IEEE Transactions on Signal Processing, vol. 44, pp. 181189, 1996.

[3] J. C. Geromel, "Optimal linear filtering under parameter uncertainty," IEEE Transactions on Signal processing, vol. 47, pp. 168-175, 1999.

[4] C. E. de Souza and A. Trofino, "An LMI approach to the design of robust $\mathscr{H}_{2}$ filters," in Advances in Linear Matrix Inequality Methods in Control, L. E. Ghaoui and S. Niculescu, Eds. SIAM, 2000, ch. 9, pp. $175-185$.

[5] J. C. Geromel, M. C. de Oliveira, and J. Bernussou, "Robust filtering of discrete-time linear systems with parameter-dependent Lyapunov functions," Proceedings of the $38^{\text {th }}$ Conference on Decision and Control, pp. 570-575, 1999.

[6] K. A. Barbosa, C. E. de Souza, and A. Trofino, "Robust $\mathscr{H}_{2}$ filtering for uncertain linear systems: LMI based methods with parametric Lyapunov function," System \& Control Letters, vol. 54, pp. 251-262, 2005.

[7] K. A. Barbosa and A. Trofino, "Robust $\mathscr{H}_{2}$ filtering for linear system with uncertain time-varying parameters," Proceedings of the $41^{\text {th }}$ IEEE Conference on Decision an Control, pp. 3883-3888, 2002.

[8] C. E. de Souza, K. A. Barosa, and A. Trofino, "Robust filtering for linear systems with convex-bounded uncertain time-varying parameters," IEEE Transactions on Automatic Control, vol. 52, pp. 1132-1138, 2007.

[9] X. Jun and X. Lihua, "An improved approach to robust $\mathscr{H}_{2}$ and $\mathscr{H}_{\infty}$ filter design for uncertain linear system with time-varying parameters," Proceedings of the $26^{\text {th }}$ Chinese Control Conference, pp. 668-672, 2007.

[10] A. Megretski and A. Rantzer, "System analysis via integral quadratic constraints," IEEE Transactions on Automatic Control, vol. 42, pp. 819-830, 1997.

[11] U. Jönsson and A. Rantzer, "Systems with uncertain parameters - timevariations with bounded derivatives," International Journal of Robust and Nonlinear Control, vol. 6, pp. 969-982, 1996.

[12] A. Helmersson, "An IQC-based stability criterion for systems with slowly varying parameters," in Proceedings of the $14^{\text {th }}$ IFAC Trienial World Congress Beijing, China, vol. 14, pp. 525-530, 1999.

[13] H. Köroğlu and C. W. Scherer, "Robust stability analysis against perturbations of smoothly time-varying parameters," Proceedings of the $45^{\text {th }}$ IEEE Conference on Decision \& Control, pp. 2895-2900, 2006.

[14] — , "Robust performance analysis for structured linear time-varying perturbations with bounded rates-of-variation," IEEE Transactions on Automatic Control, vol. 52, pp. 197-211, 2007.

[15] C. W. Scherer and I. E. Köse, "Robustness with dynamic integral quadratic constraints: An exact state space characterization of nominal stability with applications to robust estimation." Automatica, vol. 44, pp. 1666-1675, 2008.

[16] F. Paganini and E. Feron, "LMI methods for robust $\mathscr{H}_{2}$ analysis: a survey with comparisons," in In Advances in Linear Matrix Inequality Methods in Control, L. E. Ghaoui and S. Niculescu, Eds. SIAM, 1999, ch. 7, pp. 129-151.

[17] C. W. Scherer and S. Weiland, Linear Matrix inequalities in Control - Lecture Notes - Delft University of Technology, 2005.

[18] C. W. Scherer, "An efficient solution to multi-objective control problems with LMI objectives," System \& Control Letters, vol. 40, pp. 43-57, 2000.

[19] S. G. Dietz, C. W. Scherer, and H. Köroğlu, "Robust controller synthesis for disturbance filter uncertainty described by dynamic integral quadratic constraints," Proceedings of the International Federation of Automatic Control, pp. 1325-1330, 2008. 